\title{
Spectral solution methods for free-surface flow: the Rayleigh-Taylor problem
}

\author{
L. K. Forbes ${ }^{1}$
}

(Received 1 August 2008; revised 5 December 2008)

\begin{abstract}
This article describes two spectral methods for solving problems in interfacial fluid mechanics. These are illustrated here for the RayleighTaylor instability, in which a layer of heavy fluid lies above a light fluid. Disturbances to the interface between them are unstable, and grow with time. The first spectral method solves time dependent, freesurface problems in inviscid flow theory. It is capable of following the development of the interface almost to the time at which the curvature at the interface becomes infinite, and the inviscid model then ceases to be valid. A second spectral method is presented, and solves the viscous Boussinesq equations. It shows that the curvature singularity in the inviscid model is associated with regions of large vorticity at precisely these same points on the interface. Consequently, the interface rolls over at these points, forming delicate overhanging structures, leading ultimately to mixing of the two fluid layers.
\end{abstract}

http://anziamj . austms.org. au/ojs/index.php/ANZIAMJ/article/view/1393 gives this article, (c) Austral. Mathematical Soc. 2008. Published December 16, 2008. ISSN 1446-8735. (Print two pages per sheet of paper.) 


\section{Contents}

1 Introduction

C550

2 Inviscid Rayleigh-Taylor model

C552

3 Viscous Rayleigh-Taylor model

C557

4 Discussion of results

C559

5 Conclusion

C566

References

C567

\section{Introduction}

Rayleigh-Taylor flow refers to a situation in which two fluid layers lie horizontally, with a heavier fluid above a lighter one. Any disturbance to the interface between the fluids is therefore expected to be unstable and grow with time, as the heavier and lighter layers effectively seek to exchange their positions.

This classical problem was originally formulated by Rayleigh [10] and developed later by Taylor [11]. Those theories assumed ideal inviscid fluid, and performed a linearized analysis of the development of the interface profile. A sinusoidal interfacial wave was shown to grow exponentially with time. However, after some finite time linearized small amplitude theory ceases to be valid for a growing profile, and non-linear effects then become important. For inviscid fluids, it is now known that the growing interface develops a singularity in curvature within a finite time, following the work of Moore [8], for a different fluid instability (the Kelvin-Helmholtz instability). A similar analysis of the critical time for singularity formation in the Rayleigh-Taylor 
instability was presented by Baker, Caflisch and Siegel [1].

It has long been anticipated that the development of a curvature singularity at the interface, in an inviscid model of Rayleigh-Taylor flow, might be associated with interface roll-up when viscous effects are re-introduced. Krasny [7] developed a 'vortex blob' method in which the fluid interface is effectively made to have finite width in an otherwise inviscid model, and showed that, for Kelvin-Helmholtz flow, he could compute spiral waves at times beyond the critical time at which the curvature singularity was expected. However, Baker and Pham [2] demonstrated that different vortex blob methods give rise to different development of the instability, and so the reliability of these techniques in predicting the precise details of the solution beyond the critical time remains unclear.

Purely numerical solutions of the Rayleigh-Taylor instability have been undertaken by several authors, and there is now a large literature on the phenomenon of interface roll-up. Tryggvason and Unverdi [12], for example, presented numerical simulations for complex solution geometry, even in three dimensional flow, and a finite difference solution of the phenomenon in a model of turbulent flow has been given recently by Ramaprabhu et al. [9].

This article outlines a spectral method for the solution of the RayleighTaylor problem, for inviscid flow. This method has the advantage that it reduces the problem to a set of equations only at the interface, and is therefore a serious competitor to boundary integral approaches. It has the further advantage that it involves only the solution of a system of ordinary differential equations in time for the Fourier coefficients, which can be done very efficiently. Forbes, Chen and Trenham [5] first presented the technique, and Forbes and Hocking [6] later studied draining from a tank. As a check, a different spectral method is outlined for solving the Rayleigh-Taylor problem in a viscous fluid, under the Boussinesq approximation. The two sets of results are in close agreement for early times, but the curvature singularity predicted by inviscid theory is the precise trigger for interface roll-up in the viscous model, through the formation of regions of high vorticity. 


\section{Inviscid Rayleigh-Taylor model}

Consider two horizontal fluid layers in a channel of finite height. Each fluid is subject to the downward acceleration $g$ of gravity. The fluids would be stationary if the lower layer were heavier, and so it is only buoyancy effects that drive the unstable flow. This is not the classical Rayleigh-Taylor problem of Rayleigh [10] and Taylor [11], in which each fluid is of infinite vertical extent and moves vertically at some constant acceleration, but it is closely related to it, and is considered here for numerical convenience. The upper and lower fluids have density $\rho_{2}$ and $\rho_{1}$ respectively, and we are interested in the unstable situation $\rho_{2}>\rho_{1}$. A periodic disturbance of wavelength $\lambda$ exists horizontally along the interface.

Dimensionless variables are defined, and will be used from now on. All lengths are scaled relative to the quantity $\lambda / 2 \pi$, and the appropriate unit of time is $\sqrt{\lambda /(2 \pi g)}$. Speeds are measured relative to $\sqrt{g \lambda /(2 \pi)}$. In this new dimensionless formulation, the horizontal disturbances now have period $2 \pi$, and solutions are determined by three non-dimensional parameters. These are the fluid density ratio $D=\rho_{2} / \rho_{1}$ and the depths $h_{2}$ and $h_{1}$ of the upper and lower fluid depths, respectively. The situation is sketched in Figure 1.

The two fluids are assumed to be incompressible and to flow irrotationally. Consequently, velocity potentials $\phi_{2}$ and $\phi_{1}$ are constructed in fluids 2 and 1 , respectively. The fluid velocity vectors in each layer are then calculated from the gradients of the potentials, as $\left(u_{j}, v_{j}\right)=\left(\partial \phi_{j} / \partial x, \partial \phi_{j} / \partial y\right)$ in each fluid, $j=1,2$. The velocity potentials satisfy Laplace's equations

$$
\begin{array}{ll}
\nabla^{2} \phi_{1}=0 & \text { in }-h_{1}<y<\eta(x, t), \\
\nabla^{2} \phi_{2}=0 & \text { in } \eta(x, t)<y<h_{2},
\end{array}
$$

in each fluid layer.

There is no flow normal to the top and bottom walls, and this is expressed 


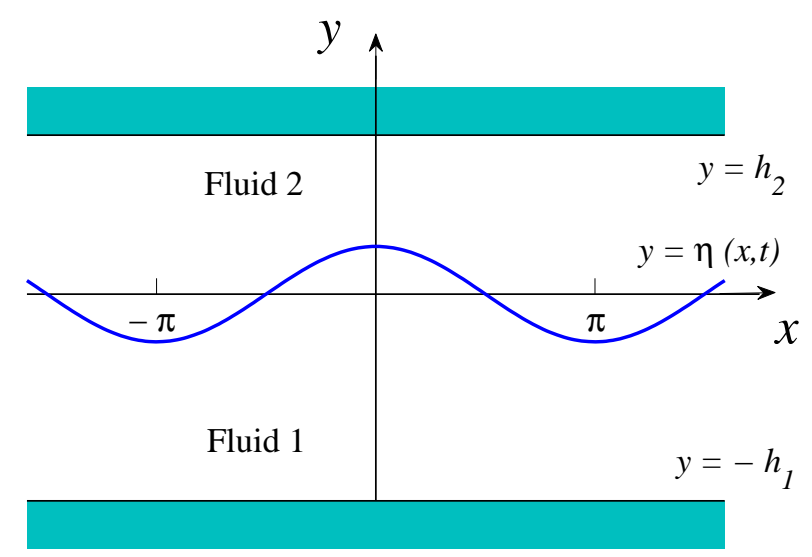

Figure 1: A sketch of the dimensionless flow configuration for the inviscid Rayleigh-Taylor problem in finite depth.

by the conditions

$$
\begin{array}{ll}
v_{1}=0 & \text { on } y=-h_{1}, \\
v_{2}=0 & \text { on } y=h_{2} .
\end{array}
$$

In addition, neither fluid can cross the interface shown in Figure 1, and this leads to the two kinematic conditions

$$
v_{j}=\frac{\partial \eta}{\partial t}+u_{j} \frac{\partial \eta}{\partial x}, \quad j=1,2, \quad \text { on } y=\eta(x, t) .
$$

Finally, since Bernoulli's equation holds as a first integral of the equations of motion in each fluid layer, imposing the dynamic condition that the fluid pressures are equal at the interface leads to the requirement

$$
\mathrm{D} \frac{\partial \phi_{2}}{\partial \mathrm{t}}-\frac{\partial \phi_{1}}{\partial \mathrm{t}}+\frac{1}{2} \mathrm{D}\left(\mathrm{u}_{2}^{2}+v_{2}^{2}\right)-\frac{1}{2}\left(\mathrm{u}_{1}^{2}+v_{1}^{2}\right)+(\mathrm{D}-1) \eta=0 \quad \text { on } \mathrm{y}=\eta(x, \mathrm{t})
$$


in non-dimensional form.

It is evident that the Laplace equations (1) and the conditions (2) are satisfied at once by velocity potentials having the forms

$$
\begin{aligned}
& \phi_{1}(x, y, t)=P_{0}(t)+\sum_{n=1}^{N} P_{n}(t) \cosh \left(n\left(y+h_{1}\right)\right) \cos (n x) \\
& \phi_{2}(x, y, t)=Q_{0}(t)+\sum_{n=1}^{N} Q_{n}(t) \cosh \left(n\left(y-h_{2}\right)\right) \cos (n x) .
\end{aligned}
$$

The upper index $\mathrm{N}$ in these sums represents the number of Fourier modes taken in the numerical solution, and should be taken as large as practicable. The time dependent Fourier coefficients in these representations (5) are to be determined. In fact, the zeroth order terms $P_{0}$ and $Q_{0}$ have no effect on the final velocity components, and we set $P_{0}(t)=0$ in the numerical scheme, and solve only for $\mathrm{Q}_{0}(\mathrm{t})$.

The interface $y=\eta(x, t)$ is now parametrized using the usual arclength $s$ defined from the relationship $\mathrm{ds}^{2}=\mathrm{dx^{2 }}+\mathrm{d \eta}^{2}$. This allows for the possibility of vertical or overhanging sections in the interface profile. We set $s=0$ at $x=0$, but the arclength has some unknown values $s= \pm \mathrm{L}(\mathrm{t})$ at the borders $x= \pm \pi$ of the periodic interval. Accordingly, it is convenient to define a new scaled arclength $\xi$ as

$$
\xi=\pi \mathrm{s} / \mathrm{L}(\mathrm{t}) .
$$

This new quantity $\xi$ now takes known values $\xi=0$ at $x=0$ and $\xi= \pm \pi$ at $x= \pm \pi$. The interface is represented in the form

$$
\begin{aligned}
& x(\xi, t)=\xi+\sum_{n=1}^{N} A_{n}(t) \sin (n \xi), \\
& \eta(\xi, t)=B_{0}(t)+\sum_{n=1}^{N} B_{n}(t) \cos (n \xi) .
\end{aligned}
$$


The time dependent Fourier coefficients in this expression are again to be determined, along with the interface half-length $\mathrm{L}(\mathrm{t})$ in equation (6).

The governing equations are now Fourier decomposed, to yield a system of equations for the time dependent coefficients. To begin, the arclength condition

$$
\left(\frac{\partial x}{\partial \xi}\right)^{2}+\left(\frac{\partial \eta}{\partial \xi}\right)^{2}=\frac{L^{2}(t)}{\pi^{2}}
$$

is integrated over a period, and the usual orthogonality relations for the trigonometric functions yield the result

$$
L(t)=\pi \sqrt{1+\frac{1}{2} \sum_{n=1}^{N} n^{2}\left[A_{n}^{2}(t)+B_{n}^{2}(t)\right]},
$$

for the unknown interface half-length $\mathrm{L}(\mathrm{t})$ in terms of the Fourier coefficients in equation (7). Following Forbes et al. [5], the arclength equation (8) is now differentiated with respect to $t$ holding $\xi$ constant, and Fourier decomposed by multiplying by basis functions $\cos (j \xi), j=1, \ldots, N$, and integrating over a period. This leads to a system of $\mathrm{N}$ ordinary differential equations involving coefficients $A_{n}(t)$ and $B_{n}(t), n=1,2, \ldots, N$.

The first kinematic condition in the system (3) is first re-written in parametric form, in terms of the scaled arclength $\xi$ in equation (6). It is then subject to Fourier decomposition as above, by multiplying by basis functions $\cos (j \xi)$ and integrating over a period. Integration by parts simplifies the algebra considerably, and the zeroth order mode yields

$$
B_{0}(t)=-\frac{1}{2} \sum_{n=1}^{N} n A_{n}(t) B_{n}(t) .
$$

It may be shown that the interface elevation, averaged across a period, equals $B_{0}(t)$ plus the term on the right hand side of equation (10). As a result, the average interface height remains zero for all times. The higher 
Fourier modes then lead to a system of $\mathrm{N}$ ordinary differential equations involving the time dependent coefficients in equation (7).

The second kinematic condition in equations (3) is actually replaced by the difference of the two, and then subject to Fourier decomposition as outlined above. It is found that the zeroth mode is satisfied as an identity, following the use of integration by parts. Higher modes are then obtained by multiplying by basis functions $\cos (j \xi), j=1,2, \ldots, N$, and integrating over a period, as before. The use of integration by parts yields an elegant algebraic identity between the Fourier coefficients. This leads to a differential algebraic system of equations for the coefficients. However, this algebraic component is avoided here simply by differentiating the algebraic identity with respect to time, giving a pure system of ordinary differential equations. The fact that such a possibility is available here represents a further advantage of this spectral approach.

Finally, the dynamic condition (4) is also subjected to similar Fourier analysis. It is occasionally convenient to add the surface tension term

$$
\sigma \kappa=\sigma \frac{\eta_{x x}}{\left[1+\eta_{x}^{2}\right]^{3 / 2}}
$$

to the left-hand side of the equation, in which $\sigma$ is a dimensionless constant representing the surface tension parameter and $\mathrm{k}$ is the curvature of the interface. Surface tension effectively regularizes the interface by penalizing high curvature, and so smoothes the interface. In terms of the arclength $\xi$, the curvature is

$$
\kappa=\frac{\pi^{3}}{L^{3}(t)}\left[x_{\xi} \eta_{\xi \xi}-x_{\xi \xi} \eta_{\xi}\right],
$$

in which $L(t)$ is the interface half-length defined in equation (9). Fourier analysis now yields a system of $\mathrm{N}+1$ differential equations for the zeroth and higher order modes.

The arclength equation, the two kinematic conditions and the dynamic condition give rise to a coupled system of $4 \mathrm{~N}+1$ ordinary differential equa- 
tions for the time dependent Fourier coefficients in equations (5) and (7). They are solved here to high accuracy using the classical fourth order RungeKutta scheme, and this has been checked against backward difference schemes. Good accuracy is obtained with $\mathbf{N}=35$ Fourier modes in equations (5) and (7), and larger values of $\mathbf{N}$ produce no change. There are integral terms involved in the Fourier decomposition of the kinematic and dynamic conditions, and these are evaluated using 301 spatial mesh points over one period of the interface, and the composite trapezoidal rule. Solutions are obtained in about 20 minutes run time on an Intel Core 2 Duo pentium $(2.1 \mathrm{GHz})$ computer.

\section{Viscous Rayleigh-Taylor model}

Here we present a spectral method for solving a simplified model of viscous flow, for the same problem as in the previous section. We use the Boussinesq approximation, in which there is only a single fluid, but the density varies smoothly with height $y$. The fluid is regarded as weakly compressible, and the interface is replaced by a narrow region of finite width in which the density changes rapidly but continuously. This follows the approach taken by Farrow and Hocking [4].

The fluid velocity components $\boldsymbol{u}$ and $v$ are calculated from a streamfunction $\psi$ in the form $(u, v)=(\partial \psi / \partial y,-\partial \psi / \partial x)$. It is also convenient to define the vorticity

$$
\zeta=\frac{\partial v}{\partial x}-\frac{\partial u}{\partial y}=-\nabla^{2} \psi,
$$

and then, by cross differentiating the momentum equations to eliminate the pressure, we obtain the familiar vorticity equation

$$
\frac{\partial \zeta}{\partial t}+u \frac{\partial \zeta}{\partial x}+v \frac{\partial \zeta}{\partial y}=-\frac{\partial \bar{\rho}}{\partial x}+\beta\left(\frac{\partial^{2} \zeta}{\partial x^{2}}+\frac{\partial^{2} \zeta}{\partial y^{2}}\right),
$$


given by Batchelor [3, page 267]. The quantity $\bar{\rho}$ is the small perturbation to the background density in the Boussinesq approximation, and it is transported according to the continuity equation

$$
\frac{\partial \bar{\rho}}{\partial t}+u \frac{\partial \bar{\rho}}{\partial x}+v \frac{\partial \bar{\rho}}{\partial y}=\gamma\left(\frac{\partial^{2} \bar{\rho}}{\partial x^{2}}+\frac{\partial^{2} \bar{\rho}}{\partial y^{2}}\right),
$$

in which the parameter $\gamma$ is a small diffusion coefficient. Similarly, $\beta$ in equation (13) is the dimensionless kinematic viscosity.

For a viscous fluid, the no-slip condition would normally be enforced at the upper and lower horizontal walls. However, since our concern is with the fluid behaviour near the interface, we simplify the problem by allowing slip at each wall, following Farrow and Hocking [4], according to the approximations

$$
v=\zeta=0 \quad \text { at } y=-h_{1}, h_{2} .
$$

By periodicity and symmetry, it is also to be expected that $u=0$ at $x= \pm \pi$. It follows from equations (15) that the appropriate spectral representation for the streamfunction $\psi$ is

$$
\psi(x, y, t)=-\sum_{n=1}^{N} \sum_{m=1}^{M} V_{m n}(t) \sin \left(\frac{m \pi\left(y+h_{1}\right)}{h_{2}+h_{1}}\right) \sin (n x) .
$$

Representations for the velocity components and vorticity are now obtained from equations (12). The density perturbation is expressed in the spectral form

$$
\begin{aligned}
\bar{\rho}(x, y, t)= & R_{00}-\sum_{m=1}^{M} R_{m 0}(t) \sin \left(\frac{m \pi\left(y+h_{1}\right)}{h_{2}+h_{1}}\right) \\
& -\sum_{n=1}^{N} \sum_{m=1}^{M} R_{m n}(t) \sin \left(\frac{m \pi\left(y+h_{1}\right)}{h_{2}+h_{1}}\right) \cos (n x) .
\end{aligned}
$$

The vorticity equation (13) and density equation (14) are decomposed spectrally. This yields a large system of $\mathrm{M}(1+2 \mathrm{~N})$ ordinary differential 
equations for the Fourier coefficients $V_{m n}(t)$ and $R_{m n}(t)$ in the representations (16) and (17). These are integrated forward in time using Runge-Kutta integration. When $M=N=25$ and a $51 \times 51$ spatial grid of points is used in the $x y$-plane, the run time is about 20 hours on an Intel Core 2 Duo pentium $(2.1 \mathrm{GHz})$ computer.

\section{Discussion of results}

We begin by considering the results of computation with the inviscid spectral method outlined in Section 2. For definiteness, we consider density ratio $\mathrm{D}=1.05$, an initial cosine wave profile with amplitude $\epsilon=0.03$, and fluid layer depths $h_{1}=h_{2}=1.5$.

Figure 2(a) illustrates the growth of the inviscid interface for a sequence of times, and the development of the instability can be seen clearly. Eventually a curvature singularity develops on the interface, and from the asymptotic estimate given by Baker et al. [1], this singularity occurs at about time $t=$ 22.45. The results in Figure 2 show that the spectral method is capable of following the interface almost completely until this critical time. The curvatures, computed from equation (11), are shown in Figure 2(b).

The corresponding calculation with the Boussinesq viscous model of Section 3 at time $t=21$ is shown in Figure 3, where it is compared with the inviscid result of Section 2 at the same time. The black dashed line is the inviscid interface. Figure 3(a) shows that there is very close agreement between the two results at this time, so that viscosity is evidently having little effect on the interface profile. However, Figure 3(b) shows that, although the vorticity is nearly zero almost everywhere in the fluid, it concentrates at precisely the two points on the interface at which the inviscid theory predicts curvature singularities. These regions of high vorticity would be expected to cause the interface to roll up at these points. 

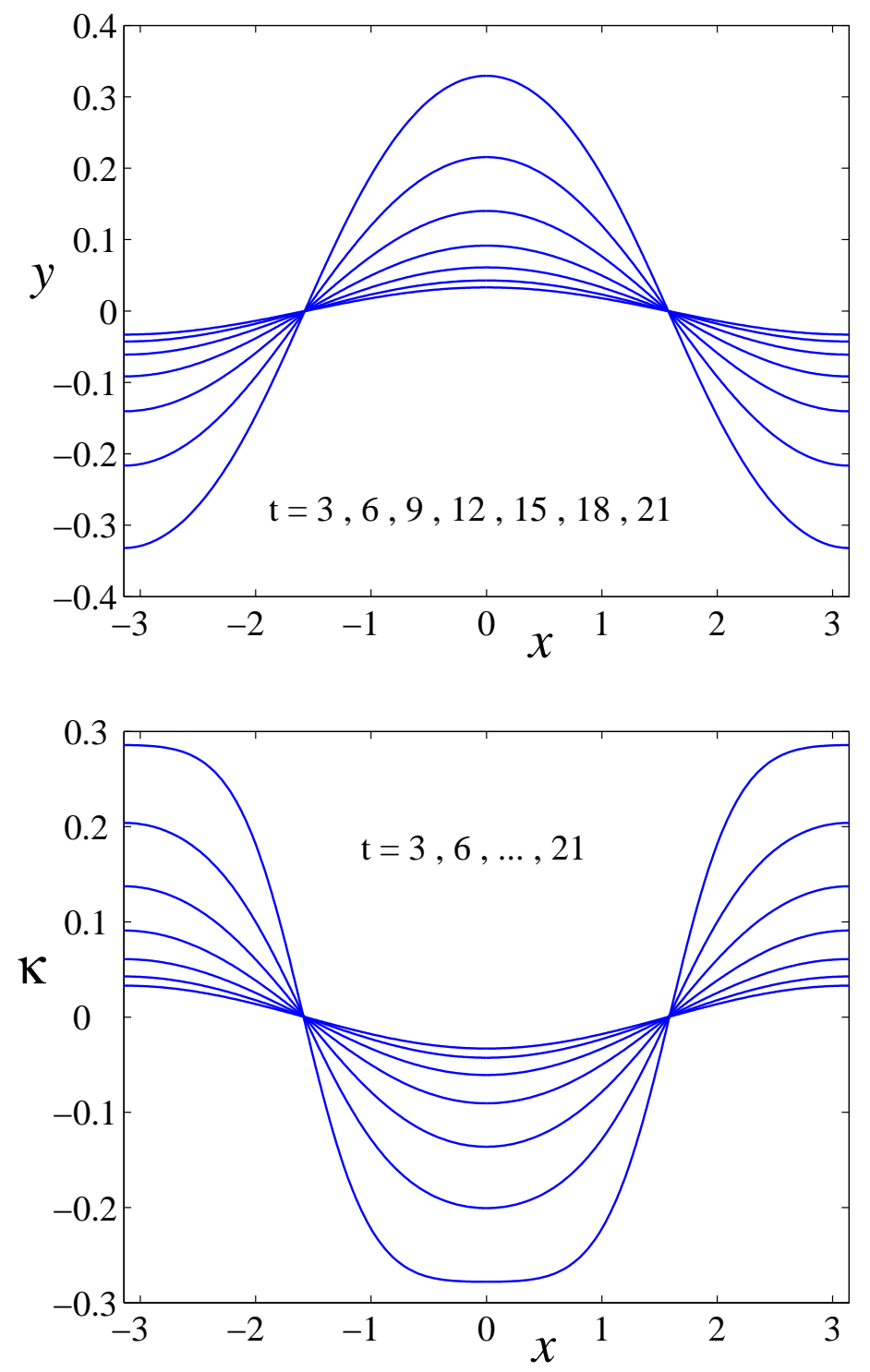

FiguRE 2: (a) Interface profiles and (b) Interface curvatures, at the sequence of times indicated, for the case $D=1.05, \epsilon=0.03, h_{1}=h_{2}=1.5$. The initial condition was a pure cosine. 

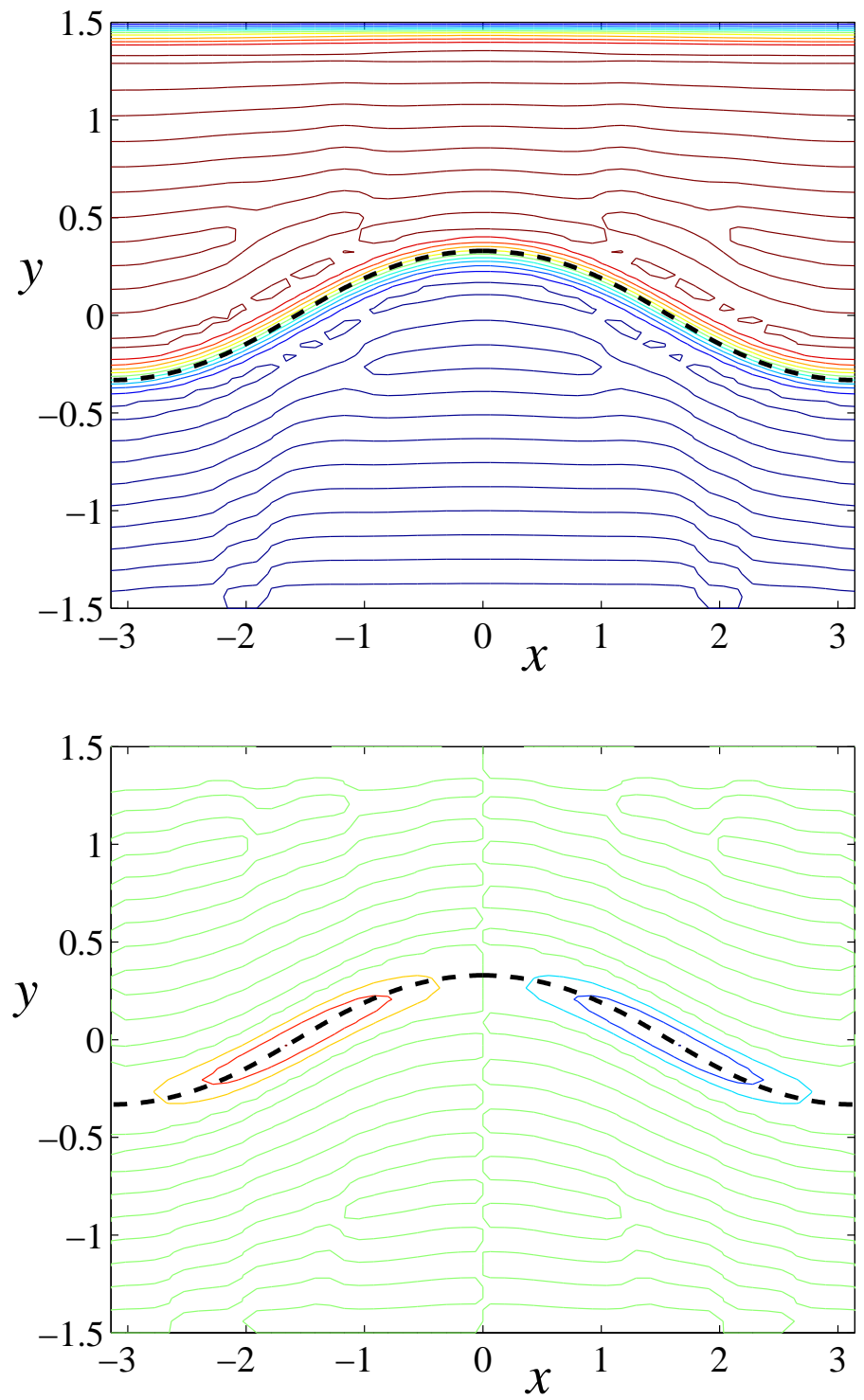

Figure 3: (a) Density contours and (b) Vorticity contours, compared against the inviscid interface profile (dashed line) at time $t=21$, for the case $\mathrm{D}=$ 1.05, $\epsilon=0.03, h_{1}=h_{2}=1.5$. The initial condition was a pure cosine. 

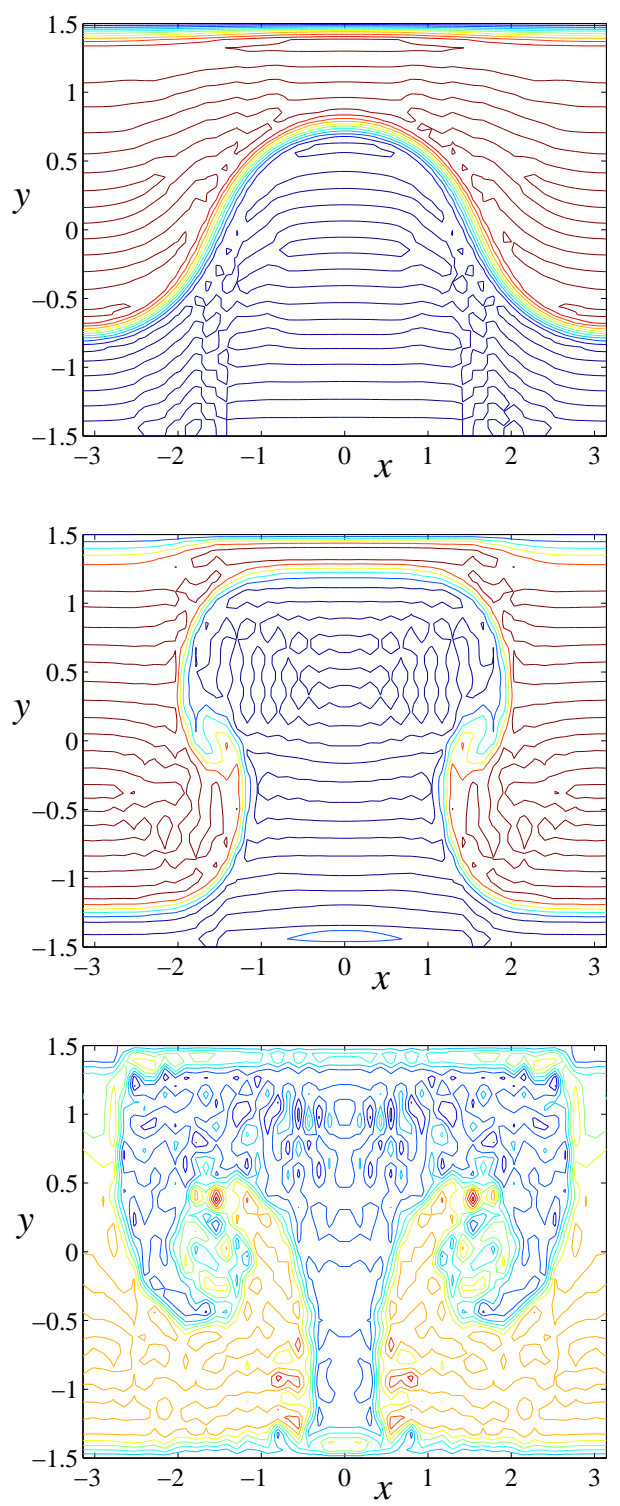

Figure 4: Development of the viscous solution at three times (a) $t=28$, (b) $t=35$ and (c) $t=42$ beyond the inviscid critical time, for the case $\mathrm{D}=1.05, \epsilon=0.03, \mathrm{~h}_{1}=\mathrm{h}_{2}=1.5$. Density contours are shown. The initial condition was a pure cosine. 
Figure 4 confirms that this is indeed what happens in the viscous solution, for times beyond the critical time at which curvature singularity is predicted by the inviscid solution. In this sequence of three solutions, the interface continues to move upwards but also rolls outwards to form an over-hanging mushroom shaped plume.

This plume is particularly evident in Figure 4(c), at the last time $t=42$. The plume of lighter fluid moving upwards has developed substantial spiral portions on the two over-hanging arms. In addition, the base has detached altogether at the bottom of the picture, and then moves upwards as time increases.

As the flow is unstable, its development is strongly affected by the choice of initial conditions. An alternative starting interface shape is considered briefly here, and has the periodic partial cosine form

$$
\eta(x, 0)= \begin{cases}\epsilon \cos (x / q) & \text { for } 0<x<q \pi \\ -\epsilon & \text { for } q \pi<x<\pi .\end{cases}
$$

In this expression (18), the constant $\mathrm{q}$ takes values $0<\mathrm{q}<1$, and the function and its first derivative are continuous at points $x= \pm \mathbf{q} \pi$.

An inviscid solution is illustrated in Figure 5, for the partial cosine profile (18) with parameter $\mathbf{q}=0.5$. Figure 5 (a) shows the growth of the interface profile, and it is clear from Figure 5(b) that there are four points along the interface at which the curvature becomes large. Consequently, it is to be expected that the viscous solution should show the interface curling up at these four points.

This is confirmed in Figure 6 in which two viscous solutions are shown at times $t=21$ and $t=28$. For the profile in Figure 6(a) at time $t=21$, the interface has clearly begun to overturn at the four points at which the curvature in Figure 5(b) is large. The viscous solution in Figure 6(b) at the later time $t=28$ shows the rolling up of the interface continuing. The downwardly moving portions of heavier fluid at the bottom of the picture 

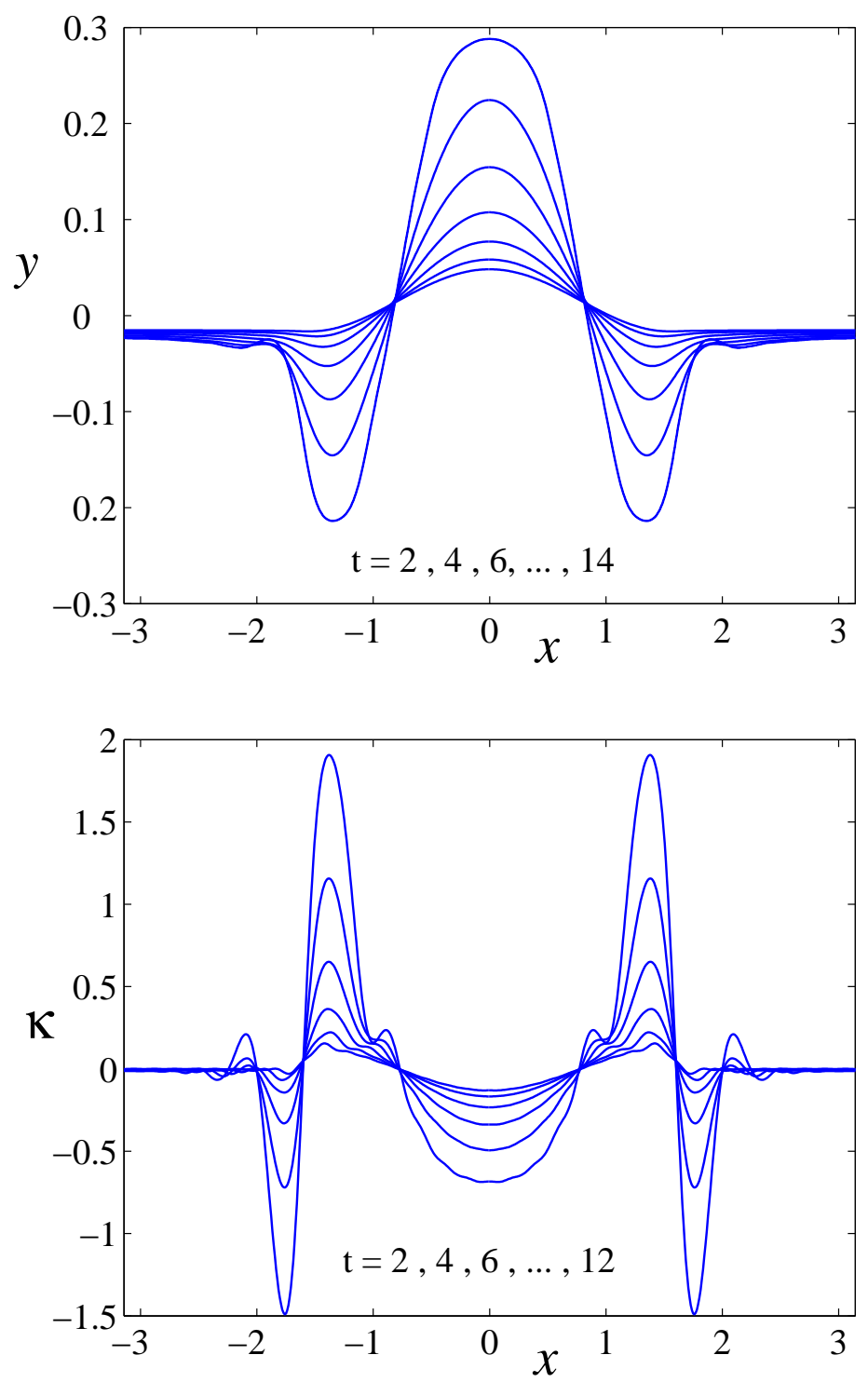

FiguRE 5: (a) Interface profiles and (b) Interface curvatures, at the sequence of times indicated, for the partial cosine initial profile. Here, $\mathrm{D}=1.05$, $\epsilon=0.03, h_{1}=h_{2}=1.5, q=0.5$ and $\sigma=2 \times 10^{-4}$. 

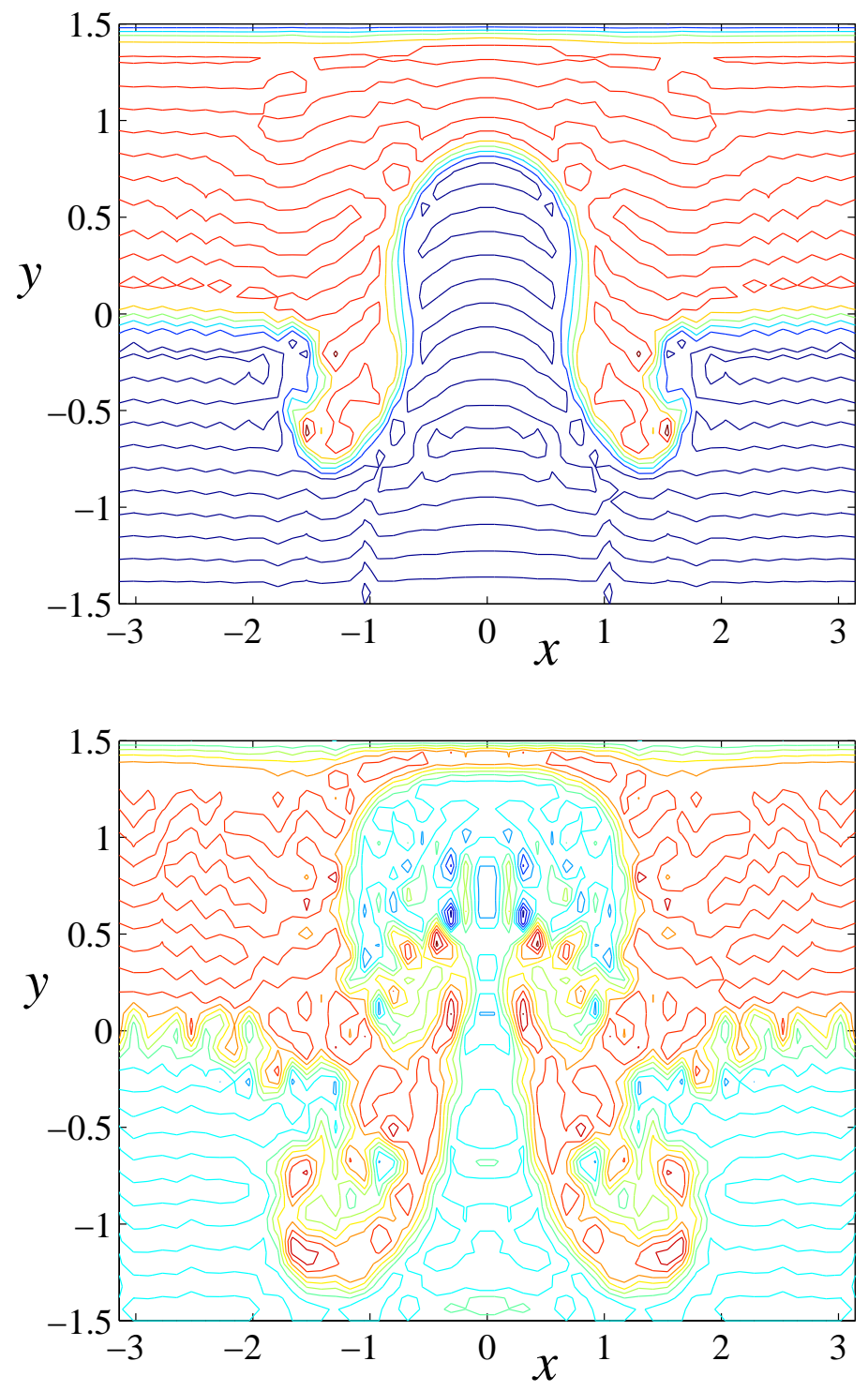

Figure 6: The viscous solution, for the partial cosine initial profile, at times (a) $t=21$ and (b) $t=28$, for the case $D=1.05, \epsilon=0.03, h_{1}=h_{2}=1.5$. Density contours are shown. 
have developed significant spiral portions, and the upwardly moving plume of lighter fluid at the centre of the diagram exhibits an over-hanging mushroom shaped profile.

\section{Conclusion}

Spectral methods for some time dependent free-surface problems in inviscid hydrodynamics are at least as efficient computationally as boundary integral methods. They allow Laplace's equation in the fluid to be satisfied identically, and the entire computational effort to focus solely on the interface itself. The evolution of the surface is followed accurately using Runge-Kutta integration to solve differential equations for the Fourier coefficients.

We illustrated this technique in the demanding situation of modelling unstable Rayleigh-Taylor flow. The method is capable of following the interface right up to the critical time at which a curvature singularity forms. These inviscid results have been compared with spectral solutions of a Boussinesq approximation for viscous flow, and the results have been shown to be in close agreement for early times. The inviscid curvature singularity triggers a local concentration of vorticity, which causes the interface to over-turn, and form over-hanging portions with spiral structures.

Acknowledgements The author is indebted to Stephen Roberts and the organizing committee for the invitation to present this material at the 2008 CTAC Conference in Canberra, Australia. 


\section{References}

[1] G. Baker, R. E. Caflisch and M. Siegel, Singularity formation during Rayleigh-Taylor instability. J. Fluid Mech., 252 (1993) 51-78. doi:10.1017/S0022112093003660 C551, C559

[2] G. R. Baker and L. D. Pham, A comparison of blob-methods for vortex sheet roll-up. J. Fluid Mech., 547 (2006) 297-316. doi:10.1017/S0022112005007305 C551

[3] G. K. Batchelor, An Introduction to Fluid Dynamics. Cambridge University Press, Cambridge (1977). C558

[4] D. E. Farrow and G. C. Hocking, A numerical model for withdrawal from a two-layer fluid. J. Fluid Mech., 549 (2006) 141-157. doi:10.1017/S0022112005007561 C557, C558

[5] L. K. Forbes, M. J. Chen and C. E. Trenham, Computing unstable periodic waves at the interface of two inviscid fluids in uniform vertical flow. J. Comput. Phys., 221 (2007) 269-287. doi:10.1016/j.jcp.2006.06.010 C551, C555

[6] L. K. Forbes and G. C. Hocking, Unsteady draining flows from a rectangular tank. Phys. Fluids, 19, 082104 (2007) 14 pages. doi:10.1063/1.2759891 C551

[7] R. Krasny, Desingularization of periodic vortex sheet roll-up. J. Comput. Phys., 65 (1986) 292-313. doi:10.1016/0021-9991(86)90210-X C551

[8] D. W. Moore, The spontaneous appearance of a singularity in the shape of an evolving vortex sheet. Proc. Roy. Soc. London A, 365 (1979) 105-119. doi:10.1098/rspa.1979.0009 C550 
[9] P. Ramaprabhu, G. Dimonte, Y.-N. Young, A. C. Calder and B. Fryxell, Limits of the potential flow approach to the single-mode Rayleigh-Taylor problem. Phys. Rev. E, 74, 066308 (2006) 10 pages. doi:10.1103/PhysRevE.74.066308 C551

[10] Lord Rayleigh, Investigation of the character of the equilibrium of an incompressible heavy fluid of variable density. Proc. London Math. Soc., 14 (1883) 170-177. C550, C552

[11] G. I. Taylor, The instability of liquid surfaces when accelerated in a direction perpendicular to their planes, I. Proc. Roy. Soc. London Ser. A, 201 (1950) 192-196. doi:10.1098/rspa.1950.0052 C550, C552

[12] G. Tryggvason and S. O. Unverdi, Computations of three-dimensional Rayleigh-Taylor instability. Phys. Fluids A, 2 (1990) 656-659. doi:10.1063/1.857717 C551

\section{Author address}

1. L. K. Forbes, School of Mathematics \& Physics, University of Tasmania, Australia.

mailto:Larry .Forbes@utas . edu . au 